\title{
Explicitly accounting for antiretroviral drug uptake in theoretical HIV models predicts long-term failure of protease-only therapy
}

\author{
Robert J. Smith? \\ Department of Mathematics and Faculty of Medicine, The University of Ottawa, 585 King Edward Ave, Ottawa, Ont., Canada K1N 6 N5
}

Received 5 July 2007; received in revised form 10 October 2007; accepted 29 November 2007

Available online 3 December 2007

\begin{abstract}
Mathematical models of HIV therapy have traditionally amalgamated the action of antiretroviral drugs, trading the complexity of the situation in favour of simpler - and hence mathematically tractable - models. However, the effects of ignoring such dynamics remain underexamined. In this paper, the traditional method of dosing (where the dose is modelled implicitly as a proportional inhibition of viral infection and production) is compared to a model that accounts for drug dynamics via explicit compartments. Four limiting cases are examined: frequent dosing of both major classes of drugs, absence of either drug, frequent dosing of one drug alone, or frequent dosing of the other drug alone. When drugs are absent, both models predict that the virus will dominate and the uninfected $\mathrm{T}$ cell counts will be low. When reverse transcriptase inhibitors are given frequently, both models predict that the virus will be theoretically eliminated and the uninfected T cell counts will be high; this is true regardless of whether the reverse transcriptase inhibitors act alone or in conjunction with protease inhibitors. However, if protease inhibitors alone are given frequently, then the implicit model predicts that the virus will be eliminated and the uninfected $\mathrm{T}$ cell counts will be high, whereas the (more realistic) explicit model predicts that the reverse situation may occur. In the latter case, critically, protease-only regimens may ultimately result in the death of the patient. It follows that the impact of drug regimens consisting only of protease inhibitors must be urgently re-examined, if such outcomes have been based on overly simplistic modelling.
\end{abstract}

(C) 2007 Elsevier Ltd. All rights reserved.

Keywords: HIV therapy; Mathematical model; Dosing intervals; Reverse transcriptase inhibitors; Protease inhibitors

\section{Introduction}

Current HIV therapy consists primarily of a combination of antiretroviral drugs, primarily drawn from two major classes, reverse transcriptase inhibitors (RTIs) and protease inhibitors (PIs) (Department of Health and Human Services, 2006). RTIs block transcription of viral RNA into DNA, preventing the infection of new cells, while PIs prevent protein cleavage in new virions, resulting in infected cells producing only noninfectious virus (Janeway et al., 2001). Historically, combination therapy has combined drugs from both classes. However, in the developing world, current antiretroviral rollout programs are staggering the release of drugs, with RTIs being used as first-line therapy and PIs held back for salvage therapy (Carpenter, 2006). In the developed world, the

E-mail address: rsmith43@uottawa.ca use of PI-only therapy has been advocated as a possible alternative to current therapy, given that the over-reliance on nucleoside RTIs has led to an increase in drug resistance (Calmy et al., 2007).

Antiretroviral drugs are given frequently, with combination therapy that may result in up to 26 doses a day, as outlined in therapy groups for men living with HIV (Prior, 2005). The effects of different drug classes have typically been aggregated in mathematical models of HIV dynamics (Calloway and Perelson, 2002; Nowak and May, 2000), with some notable exceptions (Nelson and Perelson, 2002; Perelson, 2002; Smith and Wahl, 2004). Many of these models make the assumption that the drug is widely available within the body and the (average) efficacy varies between 0 (complete drug failure) and 1 (complete inhibition of the virus). See, for example, Nelson et al. (2000, 2001), Nowak and May (2000), Perelson and Nelson (1999), and Wu and Ding (1999). This has the advantage of 
making models simpler, allowing for greater generalisability. The disadvantage is that the dynamics of drug behaviour are ignored. In particular, the drug dynamics may have a significant impact on certain outcomes; ignoring these dynamics, while appealing for model simplicity, may result in misleading conclusions.

Although there have been attempts to capture the precise dynamics of drug behaviour (Huang et al., 2003; Smith and Wahl, 2004; Wu et al., 2006), such models are significantly more complex than those which approximate the drug behaviour by a constant effectiveness. In order to determine whether including drug dynamics makes a qualitative difference, in this paper the same basic model is examined, from two perspectives. The first models drug dynamics by inhibiting viral infection and production implicitly. In this model, T cells are either susceptible or infected. This will be referred to as the implicit model. The second model approximates drug dynamics by their longterm mean value, but with the property that the magnitude of the drug values is unbounded when the dosage is large or the dosing is frequent. In this model, $\mathrm{T}$ cells may be susceptible, inhibited by one or both drugs, or infected, with or without the presence of drugs. This will be referred to as the explicit model.

This paper is organised as follows: In Section 2, the models are introduced. In Section 3, the implicit model is analysed, demonstrating the stability of equilibria when drugs are either absent or perfectly efficacious. In Section 4, the explicit model is analysed, demonstrating the long-term behaviour when drugs are either absent or in the limiting case when the dosing interval shrinks to zero. In Section 5, the outcomes of the two models are compared. In Section 6, the predictions are illustrated with numerical simulations, for more realistic drug efficacies and dosing intervals. Finally, in Section 7, the implications with regards to treatment plans are discussed, particularly the plan to treat patients using a regimen of PIs alone.

\section{The models}

Let $V_{I}$ represent infectious virus, $V_{N I}$ represent noninfectious virus, $T_{S}$ represent susceptible $\mathrm{CD}^{+} \mathrm{T}$ cells and $T_{I}$ represent infected $\mathrm{T}$ cells. Then the implicit model is given by

$$
\begin{aligned}
& \frac{\mathrm{d} V_{I}}{\mathrm{~d} t}=n_{I} \omega\left(1-\varepsilon_{P}\right) T_{I}-d_{V} V_{I}-r_{I} T_{S} V_{I}, \\
& \frac{\mathrm{d} V_{N I}}{\mathrm{~d} t}=n_{I}(1-\omega) T_{I}-d_{V} V_{N I}+n_{I} \omega \varepsilon_{P} T_{I}, \\
& \frac{\mathrm{d} T_{S}}{\mathrm{~d} t}=\lambda-r_{I}\left(1-\varepsilon_{R}\right) T_{S} V_{I}-d_{S} T_{S}, \\
& \frac{\mathrm{d} T_{I}}{\mathrm{~d} t}=r_{I}\left(1-\varepsilon_{R}\right) T_{S} V_{I}-d_{I} T_{I} .
\end{aligned}
$$

Here, $t$ is time in days, $n_{I}$ is the number of virions produced per infected cell per day, $\omega$ is the fraction of virions produced by an infected $\mathrm{T}$ cell which are infectious, $\varepsilon_{P}$ is the PI efficacy, $d_{V}$ is the rate at which free virus is cleared,
$r_{I}$ is the infection rate of noninfected $\mathrm{T}$ cells, $d_{S}$ is the noninfected $\mathrm{CD}^{+} \mathrm{T}$ cell death rate, $d_{I}$ is the infected $\mathrm{CD}^{+}{ }^{+} \mathrm{T}$ cell death rate, $\lambda$ represents a source of susceptible cells and $\varepsilon_{R}$ is the RTI efficacy. All parameters are positive, except for the drug efficacies, which satisfy $0 \leqslant \varepsilon_{P}, \varepsilon_{R}<1$.

The explicit model is given by

$$
\begin{aligned}
\frac{\mathrm{d} V_{I}}{\mathrm{~d} t}= & n_{I} \omega T_{I}-d_{V} V_{I}-r_{I} T_{S} V_{I}-r_{I} T_{P N I} V_{I}, \\
\frac{\mathrm{d} V_{N I}}{\mathrm{~d} t}= & n_{I} T_{P I}+n_{I}(1-\omega) T_{I}-d_{V} V_{N I}, \\
\frac{\mathrm{d} T_{S}}{\mathrm{~d} t}= & \lambda-r_{I} T_{S} V_{I}-d_{S} T_{S}-r_{R} T_{S} R_{a v}-r_{P} T_{S} P_{a v} \\
& +m_{R} T_{R}+m_{P} T_{P N I}, \\
\frac{\mathrm{d} T_{I}}{\mathrm{~d} t}= & r_{I} T_{S} V_{I}-d_{I} T_{I}-r_{P} T_{I} P_{a v}+m_{P} T_{P I}, \\
\frac{\mathrm{d} T_{R}}{\mathrm{~d} t}= & r_{R} T_{S} R_{a v}-d_{S} T_{R}+m_{P} T_{R P}-m_{R} T_{R}-r_{P} T_{R} P_{a v}, \\
\frac{\mathrm{d} T_{R P}}{\mathrm{~d} t}= & r_{R} T_{P N I} R_{a v}-d_{S} T_{R P}-m_{P} T_{R P}-m_{R} T_{R P}+r_{P} T_{R} P_{a v},
\end{aligned}
$$

$$
\begin{aligned}
\frac{\mathrm{d} T_{P N I}}{\mathrm{~d} t}= & r_{P} T_{S} P_{a v}-d_{S} T_{P N I}-r_{I} T_{P N I} V_{I}-r_{R} T_{P N I} R_{a v} \\
& -m_{P} T_{P N I}+m_{R} T_{R P},
\end{aligned}
$$

$\frac{\mathrm{d} T_{P I}}{\mathrm{~d} t}=r_{I} T_{P N I} V_{I}-d_{I} T_{P I}+r_{P} T_{I} P_{a v}-m_{P} T_{P I}$.

Here, additionally, $T_{R}$ represents (uninfected) cells inhibited by RTIs alone, $T_{R P}$ represents (uninfected) cells inhibited by both RTIs and PIs, $T_{P N I}$ represents uninfected cells inhibited by PIs only, $T_{P I}$ represents infected cells inhibited by PIs, $m_{R}$ is the rate at which RTIs are cleared from the intracellular compartment, $m_{P}$ is the rate at which PIs are similarly cleared, $R_{a v}$ represents RTIs and $P_{a v}$ represents PIs. All parameters are positive, except $P_{a v}$ and $R_{a v}$, which are nonnegative.

Model (2.2) is a simplification of the impulsive differential equation model introduced in Smith and Wahl (2004). In particular, the drug concentrations are given by

$$
\begin{aligned}
R_{a v} & =\frac{1}{2}\left(R\left(\tau^{+}\right)+R\left(\tau^{-}\right)\right) \\
& =\frac{R^{i}}{2}\left(\frac{1+\mathrm{e}^{-d_{R} \tau}}{1-\mathrm{e}^{-d_{R} \tau}}\right), \\
P_{a v} & =\frac{1}{2}\left(P\left(\sigma^{+}\right)+P\left(\sigma^{-}\right)\right) \\
& =\frac{P^{i}}{2}\left(\frac{1+\mathrm{e}^{-d_{P} \sigma}}{1-\mathrm{e}^{-d_{P} \sigma}}\right),
\end{aligned}
$$

where $r_{R}$ is the rate at which RTIs inhibit the T cells, $r_{P}$ is the rate at which PIs inhibit the T cells, $d_{R}$ is the rate at which RTIs are cleared, $d_{P}$ is the rate at which PIs are cleared, $R^{i}$ is the RTI dose, $P^{i}$ is the PI dose, $\tau$ is the dosing frequency of the RTIs and $\sigma$ is the dosing frequency of the PIs (At the dose times, $R^{i}$ and $P^{i}$ resemble delta functions, 
A

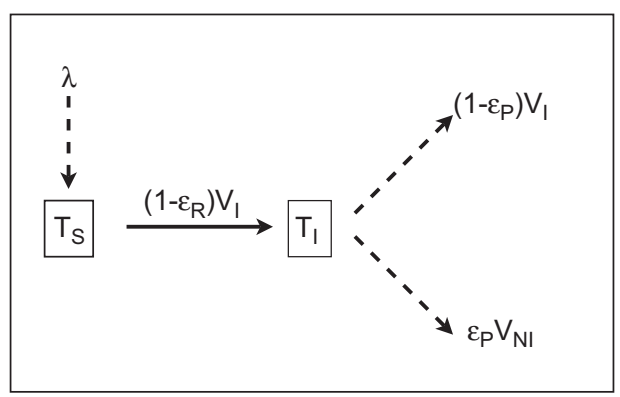

B

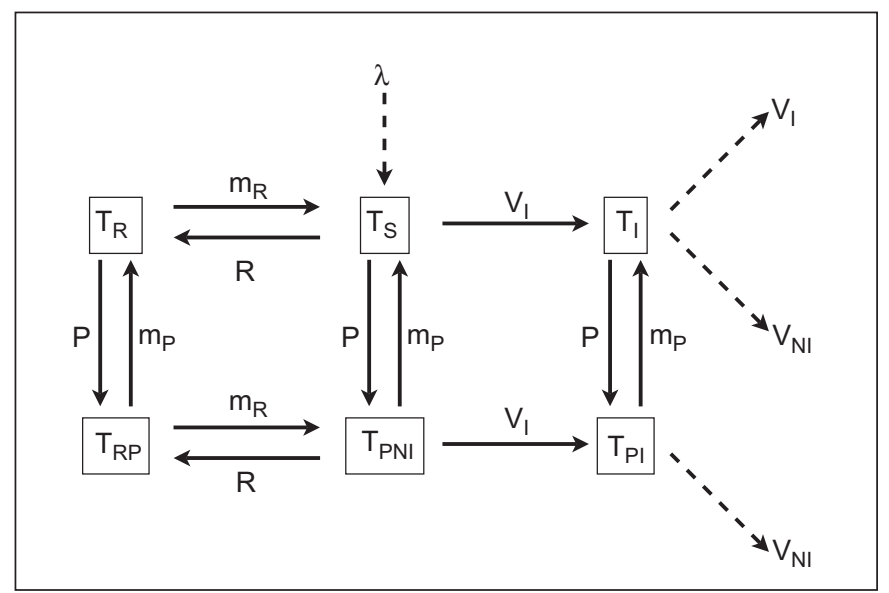

Fig. 1. Flowcharts of the models showing $\mathrm{CD}^{+} \mathrm{T}$ cell compartments. (A) The implicit model consists of susceptible $\left(T_{S}\right)$ and infected $\left(T_{I}\right)$ T cells. Susceptible cells can be infected and infected $\mathrm{T}$ cells will produce both infectious $\left(V_{I}\right)$ and noninfectious $\left(V_{N I}\right)$ virus. Drug action is implicitly modelled by the inhibition effect on new infections $\left(\varepsilon_{R}\right)$ for RTIs or production of infectious virus $\left(\varepsilon_{P}\right)$ for PIs. (B) The explicit model also consists of susceptible and infectious $\mathrm{T}$ cells, but now the compartments are divided into cells that are susceptible with no drug inhibition $\left(T_{S}\right)$, susceptible and inhibited with RTIs alone $\left(T_{R}\right)$, susceptible and inhibited with PIs alone $\left(T_{P N I}\right)$, susceptible and inhibited with both drugs $\left(T_{R P}\right)$, infected with no drug inhibition $\left(T_{I}\right)$ or infected and inhibited with PIs $\left(T_{P I}\right)$. Cells inhibited with RTIs cannot become infected, cells inhibited with PIs cannot produce infectious virus and drugs $(R, P)$ are cleared at rates $m_{R}, m_{P}$. (Note that (B) was previously published in Smith and Wahl, 2004.)

due to the impulsive conditions.). The models are illustrated in Fig. 1.

The value $R\left(\tau^{+}\right)$represents the value of the RTI on the impulsive periodic orbit immediately after a dose has been taken, while $R\left(\tau^{-}\right)$represents the value of the RTI on the impulsive periodic orbit immediately before a dose has been taken. $P\left(\sigma^{+}\right)$and $P\left(\sigma^{-}\right)$are defined similarly. Note in particular that

$$
\begin{aligned}
& \lim _{\tau \rightarrow 0} R_{a v}=\lim _{R^{i} \rightarrow \infty} R_{a v}=\infty, \\
& \lim _{\sigma \rightarrow 0} P_{a v}=\lim _{P^{i} \rightarrow \infty} P_{a v}=\infty .
\end{aligned}
$$

Thus, although the drug dynamics in the explicit model are modelled in a simplified manner (i.e., by constant values derived from the mean of the peaks and troughs of the impulsive periodic orbit from the model in Smith and Wahl, 2004), they contain the property that sufficiently frequent dosing, or a sufficiently high dose, will result in unbounded drug levels.

Models (2.1) and (2.2) are not qualitatively different; in particular, they are equivalent in the absence of drugs. Model (2.1) represents the drug effects of RTIs and PIs via the efficacy rates $\varepsilon_{R}$ and $\varepsilon_{P}$, respectively. Conversely, model (2.2) reclassifies cells inhibited by drugs into different compartments, depending on whether a cell has been inhibited by RTIs, PIs, or both.

\section{Analysis of the implicit model}

The trivial equilibrium of model (2.1) is

$\left(V_{I}, V_{N I}, T_{S}, T_{I}\right)=\left(0,0, \frac{\lambda}{d_{S}}, 0\right)$.

The nontrivial equilibrium satisfies

$$
\begin{aligned}
V_{I}= & \frac{\lambda}{d_{V} d_{I}} \frac{n_{I} \omega\left(1-\varepsilon_{P}\right)\left(1-\varepsilon_{R}\right)-d_{I}}{1-\varepsilon_{R}} \\
& -\frac{d_{S}}{r_{I}\left(1-\varepsilon_{R}\right)}, \\
V_{N I}= & \frac{n_{I}(1-\omega)+n_{I} \omega \varepsilon_{P}}{d_{V}} \\
& \times\left\{\frac{\lambda}{d_{I}}-\frac{d_{V} d_{S}}{r_{I}\left[n_{I} \omega\left(1-\varepsilon_{P}\right)\left(1-\varepsilon_{R}\right)-d_{I}\right]}\right\}, \\
T_{S}= & \frac{d_{V} d_{I}}{r_{I}\left[n_{I} \omega\left(1-\varepsilon_{P}\right)\left(1-\varepsilon_{R}\right)-d_{I}\right]}, \\
T_{I}= & \frac{\lambda}{d_{I}}-\frac{d_{V} d_{S}}{r_{I}\left[n_{I} \omega\left(1-\varepsilon_{P}\right)\left(1-\varepsilon_{R}\right)-d_{I}\right]} .
\end{aligned}
$$

Note that the nontrivial equilibrium is only defined for $\varepsilon_{R} \neq 1$. If $\varepsilon_{R}=\varepsilon_{P}=0$ (i.e., in the absence of drugs), then these equilibria are the same as those for model (2.2) in the absence of drugs (Smith and Wahl, 2004).

Since the production of infectious virions is high, we can assume $n_{I} \omega>d_{I}$. We also know that $d_{S}<d_{I}$ (Ho et al., 1995). Since $\lambda$ and $n_{I} \omega$ are both large,

$\lambda r_{I}\left[n_{I} \omega-d_{I}\right]>d_{S} d_{V} d_{I}$.

Theorem 3.1. In the absence of both drugs, the trivial equilibrium of model (2.1) is unstable and the nontrivial equilibrium is stable. Conversely, as either RTIs or PIs approach perfect efficacy, the trivial equilibrium becomes stable and the nontrivial equilibrium unstable.

Proof. The Jacobian is

$J=\left[\begin{array}{cccc}-d_{V}-r_{I} T_{S} & 0 & -r_{I} V_{I} & n_{I} \omega\left(1-\varepsilon_{P}\right) \\ 0 & -d_{V} & 0 & n_{I}(1-\omega)+n_{I} \omega \varepsilon_{P} \\ -r_{I}\left(1-\varepsilon_{R}\right) T_{S} & 0 & -r_{I}\left(1-\varepsilon_{R}\right) V_{I}-d_{S} & 0 \\ r_{I}\left(1-\varepsilon_{R}\right) T_{S} & 0 & r_{I}\left(1-\varepsilon_{R}\right) V_{I} & -d_{I}\end{array}\right]$. 
At the trivial equilibrium,

$$
\begin{aligned}
& \left.J\right|_{\left(0,0, \lambda / d_{S}, 0\right)} \\
& =\left[\begin{array}{cccc}
-d_{V}-r_{I} T_{S} & 0 & 0 & n_{I} \omega\left(1-\varepsilon_{P}\right) \\
0 & -d_{V} & 0 & n_{I}(1-\omega)+n_{I} \omega \varepsilon_{P} \\
-r_{I}\left(1-\varepsilon_{R}\right) T_{S} & 0 & -d_{S} & 0 \\
r_{I}\left(1-\varepsilon_{R}\right) T_{S} & 0 & 0 & -d_{I}
\end{array}\right] .
\end{aligned}
$$

Thus, at the trivial equilibrium,

$$
\begin{aligned}
& \operatorname{det}(J-\lambda I) \\
& =-\left(d_{V}+\lambda\right) \\
& \quad \times \operatorname{det}\left[\begin{array}{ccc}
-d_{V}-r_{I} T_{S}-\lambda & 0 & n_{I} \omega\left(1-\varepsilon_{P}\right) \\
-r_{I}\left(1-\varepsilon_{R}\right) T_{S} & -d_{S}-\lambda & 0 \\
r_{I}\left(1-\varepsilon_{R}\right) T_{S} & 0 & -d_{I}-\lambda
\end{array}\right] \\
& =\left(d_{V}+\lambda\right)\left(d_{S}+\lambda\right) \operatorname{det}\left[\begin{array}{cc}
-d_{V}-r_{I} T_{S}-\lambda & n_{I} \omega\left(1-\varepsilon_{P}\right) \\
r_{I}\left(1-\varepsilon_{R}\right) T_{S} & -d_{I}-\lambda
\end{array}\right] .
\end{aligned}
$$

This last determinant satisfies

$$
\begin{aligned}
& \lambda^{2}+\left(d_{V}+r_{I} T_{S}+d_{I}\right) \lambda+d_{V} d_{I} \\
& \quad+r_{I}\left[d_{I}-n_{I} \omega\left(1-\varepsilon_{R}\right)\left(1-\varepsilon_{P}\right)\right] T_{S}=0 .
\end{aligned}
$$

Clearly, if $\varepsilon_{R}=1$ or $\varepsilon_{P}=1$, then the constant term of the equation is positive and all eigenvalues will be negative. Thus, if either drug is perfectly efficacious, then the trivial equilibrium is stable. Conversely, if $\varepsilon_{R}=\varepsilon_{P}=0$, then (since $n_{I} \omega>d_{I}$ ) the constant term of the equation is negative. It follows that the trivial equilibrium will be unstable in the absence of both drugs.

At the nontrivial equilibrium,

$$
\begin{aligned}
& \operatorname{det}(J-\lambda I) \\
& \quad=-\left(d_{V}+\lambda\right) \\
& \quad \times \operatorname{det}\left[\begin{array}{ccc}
-d_{V}-r_{I} T_{S}-\lambda & -r_{I} V_{I} & n_{I} \omega\left(1-\varepsilon_{P}\right) \\
-r_{I}\left(1-\varepsilon_{R}\right) T_{S} & -r_{I}\left(1-\varepsilon_{R}\right) V_{I}-d_{S}-\lambda & 0 \\
r_{I}\left(1-\varepsilon_{R}\right) T_{S} & r_{I}\left(1-\varepsilon_{R}\right) V_{I} & -d_{I}-\lambda
\end{array}\right] .
\end{aligned}
$$

The characteristic equation of this last determinant is

$-\lambda^{3}-a \lambda^{2}-b \lambda+c=0$,

where

$$
\begin{aligned}
a= & d_{I}+d_{V}+r_{I} T_{S}+r_{I}\left(1-\varepsilon_{R}\right) V_{I}+d_{S}, \\
b= & d_{I} d_{V}+d_{I} r_{I} T_{S}+d_{I} r_{I}\left(1-\varepsilon_{R}\right) V_{I} \\
& +d_{I} d_{S}+d_{V} r_{I}\left(1-\varepsilon_{R}\right) V_{I}+d_{V} d_{S}+r_{I} d_{S} T_{S} \\
& -n_{I} r_{I} \omega\left(1-\varepsilon_{P}\right)\left(1-\varepsilon_{R}\right) T_{S}, \\
c= & -d_{I} d_{V} r_{I}\left(1-\varepsilon_{R}\right) V_{I}-d_{I} d_{S} d_{V}-d_{I} d_{S} r_{I} T_{S} \\
& +n_{I} \omega r_{I} d_{S}\left(1-\varepsilon_{P}\right)\left(1-\varepsilon_{R}\right) T_{S} .
\end{aligned}
$$

Substituting (3.1) and (3.3) into (3.6) gives

$$
\begin{aligned}
a= & d_{I}+d_{V}+\frac{d_{V} d_{I}}{n_{I} \omega\left(1-\varepsilon_{P}\right)\left(1-\varepsilon_{R}\right)-d_{I}} \\
& +\frac{r_{I} \lambda\left[n_{I} \omega\left(1-\varepsilon_{P}\right)\left(1-\varepsilon_{R}\right)-d_{I}\right]}{d_{V} d_{I}} .
\end{aligned}
$$

Thus,

$\lim _{\substack{\varepsilon_{R} \rightarrow 0 \\ \varepsilon_{P} \rightarrow 0}} a=d_{I}+d_{V}+\frac{d_{I} d_{V}}{n_{I} \omega-d_{I}}+\frac{\lambda r_{I}}{d_{V} d_{I}}\left[n_{I} \omega-d_{I}\right]$ $>0$,

since $n_{I} \omega>d_{I}$.

Substituting (3.1) and (3.3) into (3.7) gives

$$
\begin{aligned}
b= & d_{I} d_{V}+\frac{d_{I}^{2} d_{V}}{n_{I} \omega\left(1-\varepsilon_{P}\right)\left(1-\varepsilon_{R}\right)-d_{I}} \\
& +\frac{\lambda r_{I}}{d_{V}}\left[n_{I} \omega\left(1-\varepsilon_{P}\right)\left(1-\varepsilon_{R}\right)-d_{I}\right] \\
& +\frac{\lambda r_{I}}{d_{I}}\left[n_{I} \omega\left(1-\varepsilon_{P}\right)\left(1-\varepsilon_{R}\right)-d_{I}\right] \\
& +\frac{d_{S} d_{V} d_{I}}{n_{I} \omega\left(1-\varepsilon_{P}\right)\left(1-\varepsilon_{R}\right)-d_{I}} \\
& -\frac{n_{I} \omega\left(1-\varepsilon_{R}\right)\left(1-\varepsilon_{P}\right) d_{V} d_{I}}{n_{I} \omega\left(1-\varepsilon_{P}\right)\left(1-\varepsilon_{R}\right)-d_{I}} .
\end{aligned}
$$

Thus,

$$
\begin{aligned}
\lim _{\substack{\varepsilon_{R} \rightarrow 0 \\
\varepsilon_{P} \rightarrow 0}} b= & d_{V} d_{I}+\frac{d_{I}^{2} d_{V}}{n_{I} \omega-d_{I}}+\frac{\lambda r_{I}}{d_{V}}\left[n_{I} \omega-d_{I}\right]+\frac{d_{S} d_{V} d_{I}}{n_{I} \omega-d_{I}} \\
& +\frac{\lambda r_{I}}{d_{I}}\left[n_{I} \omega-d_{I}\right]-\frac{n_{I} \omega d_{V} d_{I}}{n_{I} \omega-d_{I}} \\
= & \frac{\lambda r_{I}}{d_{V}}\left[n_{I} \omega-d_{I}\right]+\frac{d_{S} d_{V} d_{I}}{n_{I} \omega-d_{I}}+\frac{\lambda r_{I}}{d_{I}}\left[n_{I} \omega-d_{I}\right] \\
> & 0,
\end{aligned}
$$

since $n_{I} \omega>d_{I}$.

Furthermore,

$\lim _{\substack{\varepsilon_{R} \rightarrow 1 \\ \varepsilon_{P} \rightarrow 1}} b=\lim _{\substack{\varepsilon_{R} \rightarrow 1 \\ \varepsilon_{P} \rightarrow 0}} b=\lim _{\substack{\varepsilon_{R} \rightarrow 0 \\ \varepsilon_{P} \rightarrow 1}} b=-\frac{\lambda r_{I} d_{I}}{d_{V}}-\lambda r_{I}-d_{S} d_{V}<0$.

Substituting (3.1) and (3.3) into (3.8) gives

$$
\begin{aligned}
c= & -\lambda r_{I}\left[n_{I} \omega\left(1-\varepsilon_{P}\right)\left(1-\varepsilon_{R}\right)-d_{I}\right] \\
& -\frac{d_{I}^{2} d_{S} d_{V}}{n_{I} \omega\left(1-\varepsilon_{P}\right)\left(1-\varepsilon_{R}\right)-d_{I}} \\
& +\frac{n_{I} \omega d_{S} d_{V} d_{I}\left(1-\varepsilon_{P}\right)\left(1-\varepsilon_{R}\right)}{n_{I} \omega\left(1-\varepsilon_{P}\right)\left(1-\varepsilon_{R}\right)-d_{I}} .
\end{aligned}
$$

Thus,

$$
\begin{aligned}
\lim _{\substack{\varepsilon_{R} \rightarrow 0 \\
\varepsilon_{P} \rightarrow 0}} c & =-\lambda r_{I}\left[n_{I} \omega-d_{I}\right]-\frac{d_{I}^{2} d_{S} d_{V}}{n_{I} \omega-d_{I}}+\frac{n_{I} \omega d_{S} d_{V} d_{I}}{n_{I} \omega-d_{I}} \\
& =-\lambda r_{I}\left[n_{I} \omega-d_{I}\right]+d_{S} d_{V} d_{I}<0
\end{aligned}
$$

using (3.5). 
Finally,

$\lim _{\substack{\varepsilon_{R} \rightarrow 1 \\ \varepsilon_{P} \rightarrow 1}} c=\lim _{\substack{\varepsilon_{R} \rightarrow 1 \\ \varepsilon_{P} \rightarrow 0}} c=\lim _{\substack{\varepsilon_{R} \rightarrow 0 \\ \varepsilon_{P} \rightarrow 1}} c=r_{I} \lambda d_{I}+d_{I} d_{V} d_{S}>0$.

Thus, in the absence of both drugs, the characteristic polynomial has only roots with negative real part (since $a, b>0, c<0$ ), whereas if either drug is perfectly efficacious, then the characteristic polynomial has a root with positive real part (since $b<0$ and $c>0$ ). It follows that the nontrivial equilibrium is stable in the absence of both drugs and unstable if either drug is perfectly efficacious.

Remarks. 1. Theorem 3.1 suggests that the virus could be theoretically eliminated if either drug is perfectly efficacious. However, it should be noted that this applies to free virus in plasma only; HIV has other reservoirs of virus, such as follicular dendritic cells and latently infected cells. While actual elimination is not possible using drug therapy, elimination of free virus (or, in practice, reduction below the level of detection) is obviously the most desirable situation.

2. Perelson and Nelson (1999) analysed a similar model, although they (usually) assumed $T_{S}$ was constant and did not examine the limiting effects of drugs. However, they reached similar conclusions; namely, that there is eradication if either $\varepsilon_{R}$ or $\varepsilon_{P}$ are sufficiently close to one.

\section{Analysis of the explicit model}

The following initial conditions are assumed: $V_{I}(0)=V_{0}>0, V_{N I}(0)=0, T_{I}(0)=0, T_{R}(0)=T_{R P}(0)=$ $T_{P N I}(0)=T_{P I}(0)=0$. It is also assumed that (a) $V_{0}$ is small compared to the product $n_{I} \lambda$ and (b) $T_{S}(0) \leqslant \lambda / d_{S}$, which includes the possibility that the immune system may not be operating at peak capacity when infection begins. These initial conditions correspond to the very earliest stages of infection, when the system is at the disease-free equilibrium except for a small population of infectious virus. It is therefore assumed that (1) the initial viral load is low compared to the total viral load as the infection progresses, (2) the initial (susceptible) $\mathrm{T}$ cell count is usually at the uninfected equilibrium value before infection (see Schacker et al., 1998), although the possibility that it may be less is allowed for, and (3) no drugs are taken before diagnosis.

The following lemma is straightforward, but will be used quite frequently.

Lemma 4.1. Suppose $x$ is a variable satisfying

$x^{\prime}(t)<c-q(\phi) x(t)$,

where $c$ is a constant and $q(\phi)$ is independent of $x$ and $t$. Then

(a) If $x(0)<(c / q(\phi))$, it follows that

$x(t)<\frac{c}{q(\phi)}$

for all $t$. (b) If $x(0)<(c / q(\phi))$ and $\lim _{\phi \rightarrow 0} q(\phi)=\infty$, it follows that

$$
\begin{aligned}
& x(t) \rightarrow 0 \\
& \text { as } \phi \rightarrow 0 \text { for all } t .
\end{aligned}
$$

Proof. See Smith and Wahl (2004, Lemma 4.1).

Remark. Lemma 4.1(a) also holds if the inequalities are reversed.

Let $\quad T_{\text {tot }} \equiv T_{S}+T_{R}+T_{R P}+T_{P N I}+T_{I}+T_{P I}$. Then, using Lemma 4.1,

$$
\begin{aligned}
T_{t o t}^{\prime} & =\lambda-d_{S}\left(T_{S}+T_{R}+T_{R P}+T_{P N I}\right)-d_{I}\left(T_{I}+T_{P I}\right) \\
& \leqslant \lambda-d_{S} T_{t o t},
\end{aligned}
$$

$T_{t o t}(t) \leqslant \frac{\lambda}{d_{S}}$

for all $t$, since $T_{t o t}(0) \leqslant \lambda / d_{S}$ and $d_{S}<d_{I}$. Thus, the limiting value of the total number of $\mathrm{T}$ cells with infection is less than or equal to the number of $T$ cells in the uninfected immune system. If there is no infection, then $T_{I}=T_{P I}=0$ and there is equality in (4.1). In practice, $T_{\text {tot }}(t)$ will be less than $\lambda / d_{S}$ when infection is present. By similar reasoning,

$$
\begin{aligned}
T_{t o t}^{\prime} & =\lambda-d_{S}\left(T_{S}+T_{R}+T_{R P}+T_{P N I}\right)-d_{I}\left(T_{I}+T_{P I}\right) \\
& \geqslant \lambda-d_{I} T_{t o t}, \\
T_{t o t}(t) & \geqslant \frac{\lambda}{d_{I}} .
\end{aligned}
$$

Thus,

$\frac{\lambda}{d_{I}} \leqslant T_{t o t}(t) \leqslant \frac{\lambda}{d_{S}}$.

Lemma 4.2. The infectious virions in model (2.2) satisfy $V_{I}<\frac{n_{I} \omega \lambda}{d_{S} d_{V}}$.

Proof. See Smith and Wahl (2004, Lemma 5.1).

Lemma 4.3. The susceptible T cells in model (2.2) satisfy

$T_{S}(t)>\frac{\lambda}{\alpha(\tau, \sigma)}$,

where $\alpha(\tau, \sigma) \rightarrow \infty$ as $\tau \rightarrow 0$ or $\sigma \rightarrow 0$.

Proof. Using (2.3) and Lemma 4.2,

$$
\begin{aligned}
T_{S}^{\prime} & >\lambda-r_{I} T_{S} \frac{n_{I} \omega \lambda}{d_{S} d_{V}}-d_{S} T_{S}-r_{R} T_{S} R_{a v}(\tau)-r_{P} T_{S} P_{a v}(\sigma) \\
& =\lambda-\alpha(\tau, \sigma) T_{S},
\end{aligned}
$$

where

$$
\begin{aligned}
& \alpha(\tau, \sigma)=\frac{r_{I} n_{I} \omega \lambda}{d_{S} d_{V}}+d_{S}+r_{R} R_{a v}(\tau)+r_{P} P_{a v}(\sigma) \\
& \rightarrow \infty \\
& \text { as } \tau \rightarrow 0 \text { or } \sigma \rightarrow 0 \text {. }
\end{aligned}
$$


Since $\lambda$ and $n_{I}$ are large compared to the other constants, it follows that $(\lambda / \alpha(\tau, \sigma))$ is small in general. It is thus reasonable to expect that $T_{S}(0)>(\lambda / \alpha(\tau, \sigma))$, since the body already has a sizable number of $\mathrm{T}$ cells when initially infected. Thus, by the remark following Lemma 4.1:

$T_{S}(t)>\frac{\lambda}{\alpha(\tau, \sigma)}$.

Remark. This establishes a lower limit on the number of susceptible $\mathrm{T}$ cells, which approaches zero as treatment becomes more effective.

For simplicity of notation, define $m \equiv m_{R}+m_{P}$.

Lemma 4.4. 1. If $R_{a v} \neq 0$, then, in model (2.2),

$T_{S}(t)<\frac{\lambda\left(1+\frac{m}{d_{S}}\right)}{r_{R} R_{a v}}+\delta(t, \tau, \sigma)$,

where $\delta(t, \tau, \sigma) \rightarrow 0$ as $t \rightarrow \infty$ or $\tau \rightarrow 0$ or $\sigma \rightarrow 0$.

2. If $P_{a v} \neq 0$, then, in model (2.2),

$T_{S}(t)<\frac{\lambda\left(1+\frac{m}{d_{S}}\right)}{r_{P} P_{a v}}+\delta(t, \tau, \sigma)$.

Thus, $T_{S} \rightarrow 0$ as $\tau \rightarrow 0$ or $\sigma \rightarrow 0$.

Proof. 1. Using (4.1),

$$
\begin{aligned}
T_{S}^{\prime} & <\lambda-d_{S} T_{S}-r_{R} T_{S} R_{a v}-r_{P} T_{S} P_{a v}+\frac{\lambda m_{R}}{d_{S}}+\frac{\lambda m_{P}}{d_{S}} \\
& =\lambda\left(1+\frac{m}{d_{S}}\right)-\beta(\tau, \sigma) T_{S},
\end{aligned}
$$

where

$\beta(\tau, \sigma)=d_{S}+r_{R} R_{a v}+r_{P} P_{a v}>r_{R} R_{a v}$.

Thus,

$\frac{\mathrm{d}}{\mathrm{d} t}\left(\mathrm{e}^{\beta(\tau, \sigma) t} T_{S}\right)<\lambda\left(1+\frac{m}{d_{S}}\right) \mathrm{e}^{\beta(\tau, \sigma) t}$

$T_{S}(t)<\left[T_{S}(0)-\frac{\lambda\left(1+\frac{m}{d_{S}}\right)}{\beta(\tau, \sigma)}\right] \mathrm{e}^{-\beta(\tau, \sigma) t}+\frac{\lambda\left(1+\frac{m}{d_{S}}\right)}{\beta(\tau, \sigma)}$.

Hence,

$T_{S}(t)<\delta(t, \tau, \sigma)+\frac{\lambda\left(1+\frac{m}{d_{S}}\right)}{r_{R} R_{a v}}$,

where

$\delta(t, \tau, \sigma) \equiv\left[T_{S}(0)-\frac{\lambda\left(1+\frac{m}{d_{S}}\right)}{\beta(\tau, \sigma)}\right] \mathrm{e}^{-\beta(\tau, \sigma) t}$.

Using (2.3), $\quad \beta(\tau, \sigma) \rightarrow \infty \quad$ as $\tau \rightarrow 0$ or $\sigma \rightarrow 0$ and $\delta(t, \tau, \sigma) \rightarrow 0$ as $t \rightarrow \infty$ or $\tau \rightarrow 0$ or $\sigma \rightarrow 0$. Thus, $T_{S} \rightarrow$ 0 as $\tau \rightarrow 0$ or $\sigma \rightarrow 0$.

The proof of part 2 is similar.
Remark. This establishes upper limits on the number of susceptible cells, which approach zero as treatment becomes more effective. Thus, when either RTIs or PIs (or both) are used frequently, the number of susceptible cells is driven to zero (since such cells are either infected or converted into drug-inhibited cells).

Theorem 4.1. If $R_{a v} \neq 0$, then, in model (2.2), $T_{I} \rightarrow 0$, $T_{P N I} \rightarrow 0, T_{P I} \rightarrow 0$ and $T_{R}+T_{R P} \rightarrow\left(\lambda / d_{S}\right)$ as $t \rightarrow \infty$ and $\tau \rightarrow 0$, for any fixed $\sigma$.

Proof. Using part 2 of Lemma 4.4 and (4.1),

$T_{P N I}^{\prime} \leqslant \lambda\left(1+\frac{m}{d_{S}}\right)+r_{P} \delta(t, \tau, \sigma) P_{a v}-r_{R} R_{a v} T_{P N I}+\frac{\lambda m_{R}}{d_{S}}$.

Thus, using Lemma 4.1,

$$
T_{P N I} \leqslant \frac{\lambda\left(1+\frac{m}{d_{S}}\right)+r_{P} \delta(t, \tau, \sigma) P_{a v}+\left(\frac{\lambda m_{R}}{d_{S}}\right)}{r_{R} R_{a v}} \equiv \gamma(t, \tau, \sigma)
$$

as $t \rightarrow \infty$ and $\tau \rightarrow 0$, for each fixed $\sigma$.

Using Lemma 4.2, part 1 of Lemma 4.4 and the first part of Theorem 4.1

$$
\begin{aligned}
T_{I}^{\prime}+T_{P I}^{\prime}= & r_{I}\left(T_{S}+T_{P N I}\right) V_{I}-d_{I}\left(T_{I}+T_{P I}\right) \\
\leqslant & \frac{r_{I} n_{I} \omega \lambda}{d_{S} d_{V}}\left[\frac{\lambda\left(1+\frac{m}{d_{S}}\right)}{r_{R} R_{a v}}+\delta(t, \tau, \sigma)+\gamma(t, \tau, \sigma)\right] \\
& -d_{I}\left(T_{I}+T_{P I}\right), \\
T_{I}+T_{P I} \leqslant & \frac{r_{I} n_{I} \omega \lambda}{d_{S} d_{V} d_{I}}\left[\frac{\lambda\left(1+\frac{m}{d_{S}}\right)}{r_{R} R_{a v}}+\delta(t, \tau, \sigma)+\gamma(t, \tau, \sigma)\right] \\
\rightarrow & 0
\end{aligned}
$$

as $t \rightarrow \infty$ and $\tau \rightarrow 0$, for each fixed $\sigma$.

Using Lemma 4.2 and part 1 of Lemma 4.4,

$$
\begin{aligned}
T_{S}^{\prime}+ & T_{R}^{\prime}+T_{R P}^{\prime}+T_{P N I}^{\prime}=\lambda-r_{I}\left(T_{S}+T_{P N I}\right) V_{I} \\
& -d_{S}\left(T_{S}+T_{R}+T_{R P}+T_{P N I}\right) \\
> & \lambda-\frac{r_{I} n_{I} \omega \lambda}{d_{S} d_{V}}\left[\frac{\lambda\left(1+\frac{m}{d_{S}}\right)}{r_{R} R_{a v}}+\delta(t, \tau, \sigma)+\gamma(t, \tau, \sigma)\right] \\
& -d_{S}\left(T_{S}+T_{R}+T_{R P}+T_{P N I}\right) .
\end{aligned}
$$

Using a similar argument to the proof of Lemma 4.4,

$$
\begin{aligned}
& T_{R}+T_{R P} \\
& >\frac{\lambda}{d_{S}}-\frac{r_{I} n_{I} \lambda}{d_{S}^{2} d_{I}}\left[\frac{\lambda\left(1+\frac{m}{d_{S}}\right)}{r_{R} R_{a v}}+\delta(t, \tau, \sigma)+\gamma(t, \tau, \sigma)\right]
\end{aligned}
$$




$$
\begin{aligned}
& -T_{S}-T_{P N I}+\left\{T_{S}(0)-\frac{\lambda}{d_{S}}+\frac{r_{I} n_{I} \lambda}{d_{S}^{2} d_{I}}\right. \\
& \left.\times\left[\frac{\lambda\left(1+\frac{m}{d_{S}}\right)}{r_{R} R_{a v}}+\delta(t, \tau, \sigma)+\gamma(t, \tau, \sigma)\right]\right\} \mathrm{e}^{-d_{S} t}
\end{aligned}
$$

$\rightarrow\left(\lambda / d_{S}\right)$ as $t \rightarrow \infty$ and $\tau \rightarrow 0$, for any fixed $\sigma$, using the first part of this proof and Lemma 4.4.

Remark. Thus, if RTIs are taken with sufficient frequency (with or without PIs), cells inhibited with RTIs do the work of maintaining the immune system at pre-infection levels and all other cells are driven to zero.

\section{Comparison of the implicit and explicit model}

For the purposes of comparison with the explicit model, it is assumed that the efficacy of the drugs is directly related to the frequency of drug dosing; that is, as the frequency of doses approaches infinity, the efficacy approaches $100 \%$.

In the absence of both drugs, the implicit and explicit models are equivalent. In this case, both models predict that the disease-free equilibrium is unstable and the endemic equilibrium is stable. It follows that the virus dominates in the absence of drugs.

When both drugs are taken frequently, Theorems 3.1 and 4.1 predict that the virus will be eliminated and the infected $\mathrm{T}$ cell count will be low.

When PIs are absent, but RTIs are taken frequently, Theorems 3.1 and 4.1 also predict that the virus will be eliminated and the infected $\mathrm{T}$ cell count will be low.

However, when RTIs are absent, but PIs are taken frequently, the outcome may not be consistent. In particular, Theorem 3.1 always predicts that the diseasefree equilibrium will be stable and the endemic equilibrium will be unstable in the implicit model. For the explicit model in this case, $T_{S} \rightarrow 0$ (from Lemma 4.4) and $T_{R P}=T_{R}=0$ (since the RTIs are absent). Applying (4.2), we have

$\frac{\lambda}{d_{I}} \leqslant T_{I}+T_{P N I}+T_{P I} \leqslant \frac{\lambda}{d_{S}}$.

However, typically $1 / d_{I} \ll 1 / d_{S}$. Let $\mu_{0}$ denote the minimum number of $\mathrm{T}$ cells required to maintain the immune system. If $\mu_{0}$ satisfies

$$
\frac{\lambda}{d_{I}} \leqslant \lim _{t \rightarrow \infty}\left(T_{I}+T_{P N I}+T_{P I}\right)<\mu_{0}<\frac{\lambda}{d_{S}},
$$

then there is no dosing schedule that will sustain a healthy immune system. That is, if the number of nonzero $\mathrm{T}$ cells is too low, then the effect of treatment with PIs, no matter how frequently taken or how large the dose is, ultimately may be no better than no treatment at all. Thus, in this case, the outcome depends on the model.

The results are summarised in Table 1.

\section{Numerical simulations}

Section 4 does not demonstrate what happens as $\sigma \rightarrow 0$ if $R_{a v}=0$ and $P_{a v} \neq 0$. In this case, the result may be identical to the implicit model (namely, eradication), it might satisfy (5.1) (in which case treatment with PIs is effectively similar to no treatment at all) or there may be a limiting value that exceeds $\mu_{0}$ but is still low (see Calloway and Perelson, 2002). Consequently, the outcome must be illustrated numerically.

While drugs are never perfectly efficacious, results in Sections 3 and 4 are an idealisation of the case where one or both drugs are highly efficacious. In this section, the results are illustrated for realistic parameters, where the drugs are moderately efficacious. The parameters describing $\mathrm{T}$ cell and virus dynamics are largely straightforward and were taken from the literature; the reader is referred to Haase et al. (1996), Nelson and Perelson (2002), and Wahl and Nowak (2000) for details and to the figure legends for specific values (Figs. 2 and 3 ).

To illustrate the differences in the implicit versus the explicit models, PI-only therapy was simulated. In Fig. 2, the PI efficacy was $75 \%$. Even this moderate efficacy led to low viral load for infectious virus (approximately

\begin{tabular}{|c|c|c|c|c|}
\hline RTI & PI & Implicit dosing & Explicit dosing & Result \\
\hline Infrequent & Infrequent & $\begin{array}{l}\text { Infected } \mathrm{T} \text { cell counts high, } \\
\text { Uninfected } \mathrm{T} \text { cell counts low }\end{array}$ & $\begin{array}{l}\text { Infected } \mathrm{T} \text { cell counts high, } \\
\text { Uninfected } \mathrm{T} \text { cell counts low }\end{array}$ & Virus dominates \\
\hline Frequent & Frequent & $\begin{array}{l}\text { Infected } \mathrm{T} \text { cell counts low, } \\
\text { Uninfected } \mathrm{T} \text { cell counts high }\end{array}$ & $\begin{array}{l}\text { Infected } \mathrm{T} \text { cell counts low, } \\
\text { Uninfected } \mathrm{T} \text { cell counts high }\end{array}$ & Virus eliminated \\
\hline Frequent & Infrequent & $\begin{array}{l}\text { Infected } T \text { cell counts low, } \\
\text { Uninfected } T \text { cell counts high }\end{array}$ & $\begin{array}{l}\text { Infected } T \text { cell counts low, } \\
\text { Uninfected } T \text { cell counts high }\end{array}$ & Virus eliminated \\
\hline Infrequent & Frequent & $\begin{array}{l}\text { Infected } \mathrm{T} \text { cell counts low, } \\
\text { Uninfected } \mathrm{T} \text { cell counts high }\end{array}$ & $\begin{array}{l}\text { Infected } \mathrm{T} \text { cell counts may be high, } \\
\text { Uninfected } \mathrm{T} \text { cell counts may be low }\end{array}$ & Depends on method! \\
\hline
\end{tabular}

Table 1

Comparison of implicit and explicit dosing for both major classes of drugs

If both drugs are taken infrequently, then the virus dominates. If RTIs are taken sufficiently frequently, then the virus is theoretically eliminated, regardless of PI frequency. However, if PIs are taken frequently, but RTIs are taken infrequently, then the implicit and explicit models may produce different results. 

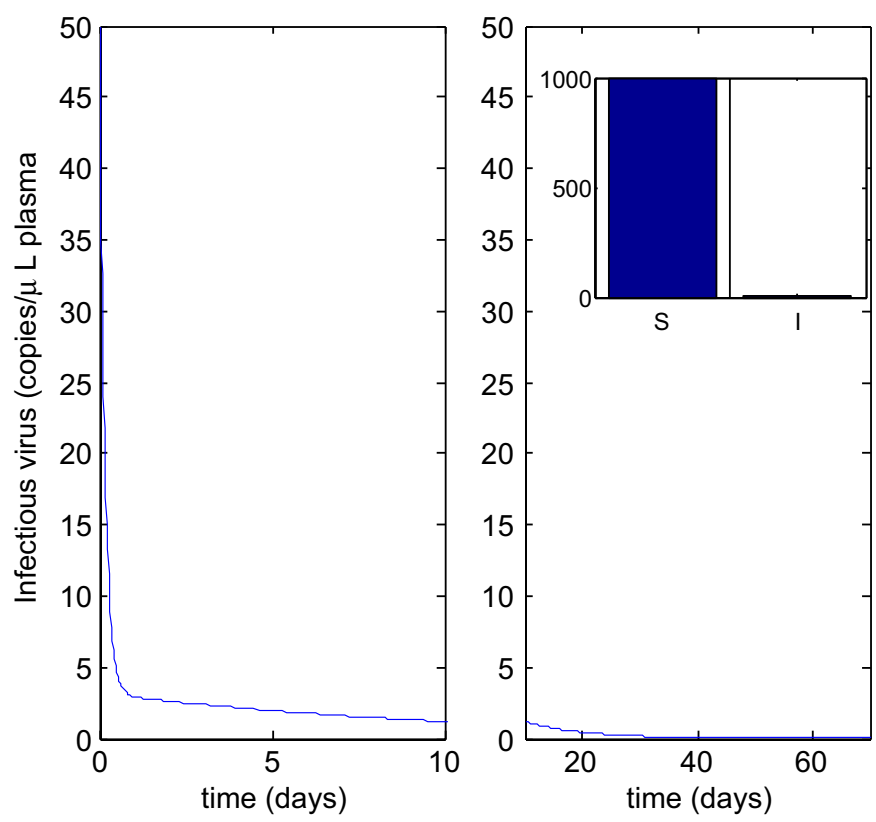

Fig. 2. PI-only therapy, using the implicit model, with efficacy $\varepsilon_{P}=0.75$. Other parameters were $n_{I}=62.5 \mathrm{day}^{-1} \quad \omega=0.05, \quad d_{V}=3 \mathrm{day}^{-1}$, $r_{I}=0.0032 \mathrm{day}^{-1}, \lambda=100$ cells $\mu \mathrm{L}^{-1} \mathrm{day}^{-1}, \varepsilon_{R}=0, d_{S}=0.1 \mathrm{day}^{-1}, d_{I}=$ $0.5 \mathrm{day}^{-1}$ (taken from Smith and Wahl, 2004 where appropriate). Initial conditions were $V_{I}(0)=50$ virions $\mu \mathrm{L}^{-1}, T_{S}(0)=1000$ cells $\mu \mathrm{L}^{-1}$ and all other initial conditions zero. In this case, high-efficacy PI-only therapy leads to theoretical elimination of the virus. Inset: equilibrium $\mathrm{CD}^{+} \mathrm{T}$ cell counts are high for susceptible and low for infected cells.

0.0043 copies $\mu \mathrm{L}^{-1}$ ) and a high $\mathrm{T}$ cell count for noninfected cells (approximately 999.2 cells $\mu \mathrm{L}^{-1}$, compared to a baseline of 1000 cells $\mu \mathrm{L}^{-1}$ for the uninfected patient).

In Fig. 3, the same parameters were used where equivalent, and the PI was given three times daily (the maximum daily dosage for any single drug or fixed-dose combination; see Calmy et al., 2007), resulting in a value of $P_{a v}=6.57$. Unlike the implicit model, the infectious viral load was significant (approximately 130 copies $\mu \mathrm{L}^{-1}$, similar to the viral load without treatment) and the uninfected $\mathrm{T}$ cell counts were low (approximately 200 cells $\mu \mathrm{L}^{-1}$ ).

Fig. 4 demonstrates how the stable viral load in the implicit model depends on the efficacy of the drugs. The nontrivial steady state is positive for a range of values of $\varepsilon_{R}$ and $\varepsilon_{P}$, but as either $\varepsilon_{R} \rightarrow 1$ or $\varepsilon_{P} \rightarrow 1$, the nontrivial steady state exchanges stability with the trivial steady state and thereafter becomes negative.

Fig. 5 demonstrates how the stable viral load in the explicit model depends on the mean concentration of the drugs. In this case, the virus is eliminated as $R_{a v}$ increases, regardless of whether PIs are present or not. However, if RTIs are absent, then the virus remains at untreated levels, even if $P_{a v}$ becomes large.

\section{Discussion}

Implicitly modelling the uptake of antiretroviral drugs may result in an overconfidence in the ability of PI-only

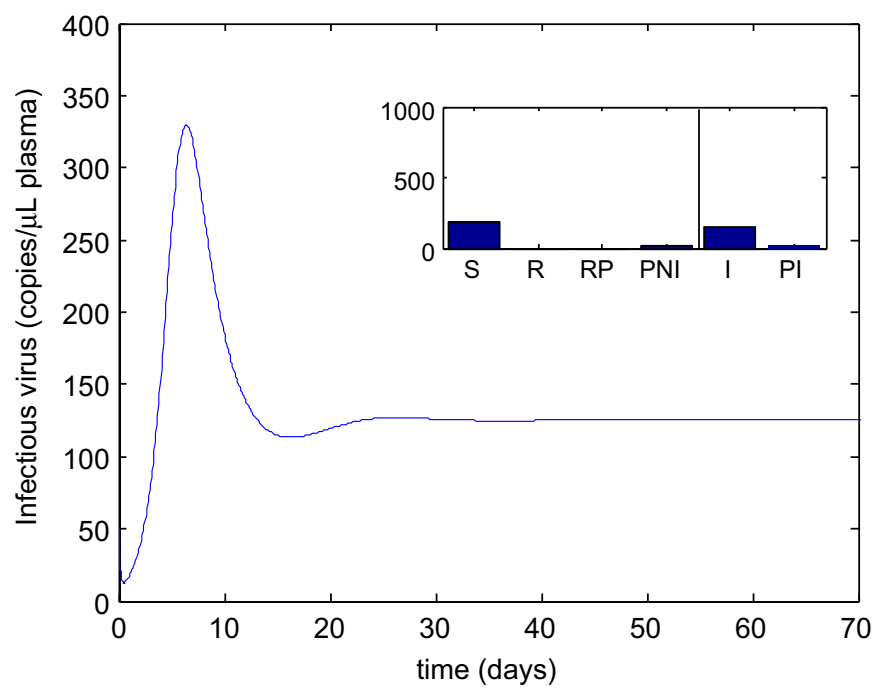

Fig. 3. PI-only therapy, using the explicit model. All equivalent parameters were as in Fig. 2. Remaining parameters were $r_{P}=$ $0.127 \mu \mathrm{M}^{-1} \mathrm{day}^{-1}, \quad r_{R}=56.1 \mu \mathrm{M}^{-1} \mathrm{day}^{-1}, m_{R}=4.16 \mathrm{day}^{-1}, m_{P}=8.52$ day $^{-1}, d_{P}=8.32$ day $^{-1}, P^{i}=11.6 \mu \mathrm{M}$ (taken from Smith and Wahl, 2004), resulting in a mean PI concentration value of $P_{a v}=6.57$. Initial conditions were as in Fig. 2. Unlike Fig. 2, high-frequency PI-only therapy does not lead to elimination of the virus. Inset: equilibrium $\mathrm{CD}^{+} \mathrm{T}$ cell counts are low for susceptible and infected cells and zero for all other cells.

therapy to combat HIV. Altering the traditional models only slightly, to include the explicit compartments of druginhibited $\mathrm{T}$ cells, results in a qualitative change in outcome for this line of therapy. Whereas RTIs prevent infection of a cell (whether used in conjunction with PIs or alone), PIs allow a cell to become infected. When the RTI wears off, the cell can only return to a noninfected state, but when the PI wears off, the cell may have already become infected. This is a consequence of the RTI and PI drug mechanisms. In particular, if RTIs are absent, then the only nonzero, noninfected cells are $P_{N I}$, which are themselves entirely susceptible to infection.

Intuitively, for the implicit model, as the efficacy of PIs approach 1 (in the absence of RTIs), the production of infectious virions (and hence infected $\mathrm{T}$ cells) is significantly reduced, but the susceptible cells are not driven towards zero. Conversely, for the explicit model, as PI dosing approaches infinity (in the absence of RTIs), most T cells become inhibited with the PI, but it only takes one virus particle to infect them. Once infected, these cells will oscillate between cells that produce infectious virions and cells that produce noninfectious virions, depending on the uptake and waning rates of the PI. This drives the noninfected cells towards zero. Once infected, the death rate of a such a cell — whether inhibited with PIs or not-is high. It follows that highly efficacious PI-only therapy may result in an abundance of susceptible cells in the implicit model, but an abundance of infected cells in the explicit model.

Thus, the traditional (implicit) method of modelling the efficacy of drugs may lead to an overconfidence in the 


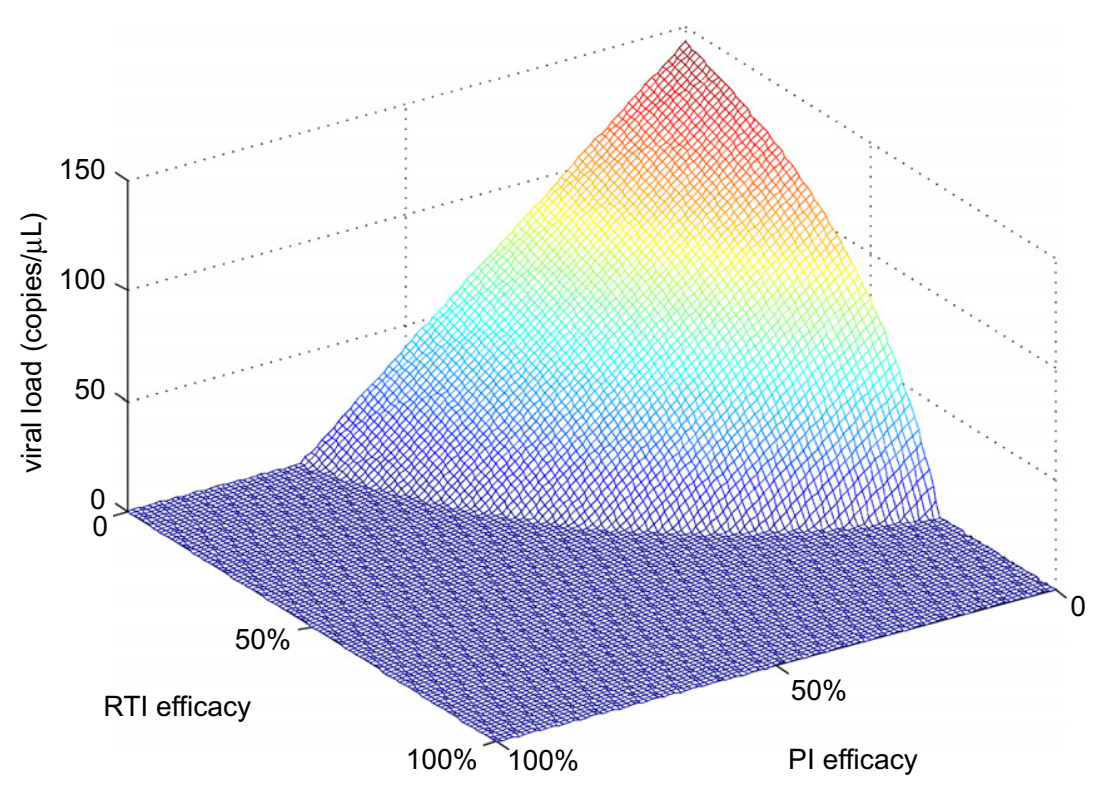

Fig. 4. Steady state viral load for the implicit model, as the efficacy of each drug varies. If both drugs are nonefficacious, then the nontrivial equilibrium is stable and the viral load is high. As the efficacy of each drug rises, the magnitude of the stable viral load decreases until the virus is eliminated. There is a critical value of each efficacy where a transcritical bifurcation occurs; at these points, the nontrivial equilibrium loses its stability and the trivial equilibrium becomes stable. The viral load at this point coalesces with the disease-free steady state; thereafter, the nontrivial viral load is negative. Parameters are as in Fig. 2. Note that this graph measures only the infectious viral load.

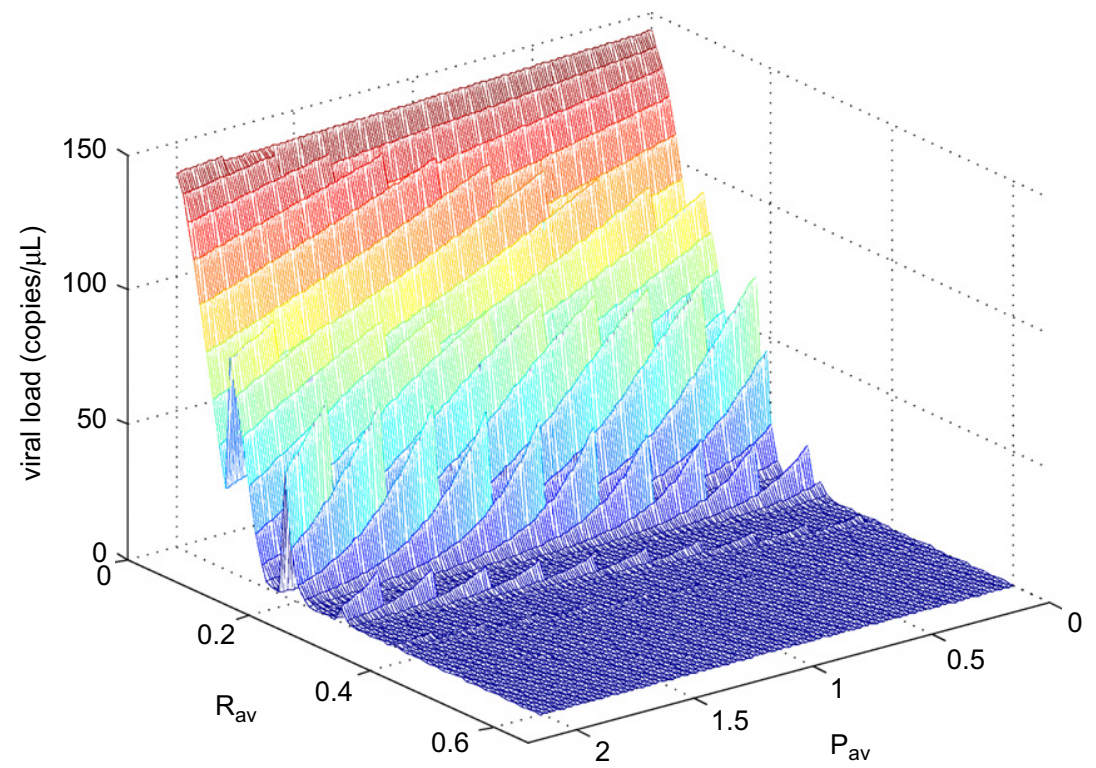

Fig. 5. Steady state viral load for the explicit model, as the mean concentration of each drug varies. If both drugs are absent, then the nontrivial equilibrium is stable and the viral load is high. As the concentration of RTIs increases, the magnitude of the stable viral load decreases until the virus is eliminated; this occurs whether PIs are present or absent. However, if RTIs are absent, the resulting viral load will be high, regardless of PI concentration and in contrast to Fig. 4. Parameters are as in Fig. 3. Note that this graph measures only the infectious viral load. (The jagged edges are due to limitations in the numerical software.)

ability of PIs to deal with the virus. While it is possible to choose parameters to ensure that PI-only therapy does lead to theoretical elimination of the virus in the explicit model (see Smith and Wahl, 2004), PI-only therapy will not eliminate the virus in all cases and, in fact, may not lead to $\mathrm{CD}^{+} \mathrm{T}$ cell counts much larger than that of no therapy at all (Figs. 3 and 5). Critically, this means that the traditional model predicts a long-term beneficial outcome for the patient, whereas the explicit model predicts that such therapy may ultimately kill the patient.

Mathematically, the implicit model determines the stability, or not, of the trivial (disease-free) equilibrium, 
compared to the endemic equilibrium. Conversely, in the explicit model, the trivial equilibrium is always unstable, for all realistic parameters, but when $\tau \rightarrow 0$, a new, stable, disease-free equilibrium (with $T_{S}=0$ ) is approached. However, as $\sigma \rightarrow 0$, no such disease-free equilibrium may be approached. Thus, while results in Section 3 are independent of parameter estimates, results in Section 4 are independent of parameter estimates only when RTIs are involved (see Theorem 4.1). If RTIs are absent, then results depend enormously on the choice of parameters; while sample parameters from the literature illustrate treatment failure (Section 6), other parameter choices will lead to treatment success (see Smith and Wahl, 2004 for more discussion).

While neither model is an exact representation of the drug dynamics in question, the explicit model captures more of the reality of the situation than the implicit model and approximates more complex models from the literature. The two models are equivalent in the absence of drugs and have similar outcomes when the RTI is included (with or without the presence of the PI), but they produce qualitatively different results when the PI acts alone. Since the explicit model captures more (although not all) of the dynamics, this suggests that models that explicitly account for the impact of drug dynamics on $\mathrm{T}$ cells are more reliable predictors of therapy outcomes.

Even with a relatively low efficacy of $75 \%$, the implicit model predicts that PI-only therapy will lead to near-total reduction in the viral load (less than 0.0043 copies $\mu \mathrm{L}^{-1}$ ), with high noninfected $\mathrm{CD}^{+} \mathrm{T}$ cell counts (Fig. 2). However, the same basic model using the same parameters but with explicit drug dynamics produced high viral load (approximately 130 copies $\mu \mathrm{L}^{-1}$ ) and low $\mathrm{CD} 4^{+}$counts for both infected and noninfected cells (Fig. 3). Fortunately, PI-sparing therapy has recently been advocated, in both the developing and developed world (Moyle, 2003). In particular, RTI-only therapy is underway in antiretroviral rollout programs in Africa, with PIs held back for secondline therapy (Carpenter, 2006).

These theoretical predictions may appear to be at odds with clinical evidence that PIs control HIV more effectively (Ghani et al., 2001). This is because this result pertains to all possible drugs which either prevent infection or prevent virion production and compares these two strategies of defense, in the absence of resistance. The effects of resistance should not be discounted, but the development of new drugs allows for the fact that mutation-resistant cocktails may now consist of numerous RTIs, numerous PIs or a combination of both. Side effects are also an important issue (Krakovska and Wahl, 2007; Moyle, 2003) that may lead to one regimen being chosen over another.

Preliminary experimental studies of PI-only therapy have been carried out in the developed world (Calmy et al., 2007) to reduce drug resistance in the short term, but such therapy has not yet been approved. In that study, 42 patients taking one or two PIs were monitored for 105 weeks as part of a larger cohort of patients assessing virological and immunological safety and activity of nucleoside-RTI-sparing regimens. Other studies have assessed preliminary 24-week trials, demonstrating that immune reconstitution and median viral load decreased (Staszewski et al., 2003a, b; Raguin et al., 2004), although these studies were mostly performed on subjects who had a heavy history of treatment before undertaking this regimen. Such experiments were devised only for short time periods and did not assess the long-term impact of PI-only therapy. Furthermore, it should be stressed that model (2.2) does not necessarily predict treatment failure for PI-only therapy, only that it is a possibility, whereas model (2.1) does not allow for such a possibility.

The explicit model examined here is a continuous version of the previously published model in Smith and Wahl (2004). This was chosen to deliberately avoid the use of impulsive differential equations, partly for clarity of argument and partly to illustrate the robustness of the results with respect to model choice (thus, the implicit model here also differs slightly from similar models in the literature; see Perelson and Nelson (1999), Wu and Ding (1999), Nelson et al. (2001, 2000), and Nowak and May (2000)). The difference lies in the structuring of the druginhibited cells via compartment modelling, compared to parameter variation in the implicit model. The inclusion of the $-r_{I} T_{S} V_{I}$ term accounts for the loss of virions due to infection; however, it makes little overall difference in the qualitative results. While the analysis in Section 4 is similar to the analysis in Smith and Wahl (2004), the model was simplified to a continuous version to (a) avoid the complications added by imposing discontinuities into the model and (b) examine the underlying dynamics in the model. Thus, the implicit and explicit models are both new and the analysis of each model is also new, although similar analyses of similar models have also been carried out in other works.

Generalising the explicit model, similar results will occur for any model that has unbounded drug levels as either the dosage or dosing frequency increases without bound and where the susceptible cells are driven towards zero, but cells inhibited with RTIs (both with and without PIs) approach the levels in the uninfected immune system. In such cases, RTIs could theoretically eliminate the virus, with or without PIs. Conversely, PIs alone may not control the virus without further restrictions on the parameters.

In summary, mathematical models of HIV should account for the explicit dynamics of $\mathrm{T}$ cells. In particular, the clinical impact of PI-only therapy must be urgently reexamined to determine long-term therapy outcomes, if such outcomes have been based on overly simplistic modelling.

\section{Acknowledgements}

The author is grateful to Shoshana Magnet, Ben Aggarwala, Beni Sahai, Jennifer Smith, Shanta Varma, Frithjof Lutscher, Benoit Dionne, Barbara Smith, Greg Smith and Marco Llamazares for technical discussions, 
and acknowledges three reviewers, whose comments helped improve the manuscript. Support from an NSERC Discovery Grant is gratefully acknowledged.

\section{References}

Calloway, D.S., Perelson, A.S., 2002. HIV-1 infection and low steady state viral loads. Bull. Math. Biol. 64, 29-64.

Calmy, A., Petoumenos, K., Lewden, C., Law, M., Bocquentin, F., Hesse, K., Cooper, D., Carr, A., Bonnet, F., 2007. Combination antiretroviral therapy without a nucleoside reverse transcriptase inhibitor: experience from 334 patients in three cohorts. HIV Med. 8, 171-180.

Carpenter, C., 2006. Universal access to antiretroviral therapy: when, not if. Clin. Infect. Dis. 42, 260-261.

Department of Health and Human Services, 2006. Guidelines for the use of antiretroviral agents in HIV-1-infected adults and adolescents $\langle$ http:// img.thebody.com/hivatis/pdfs/adult_guide.pdf) (accessed December 26, 2007).

Ghani, A.C., Henley, W.E., Donnelly, C.A., Mayer, S., Anderson, R.M., 2001. Comparison of the effectiveness of non-nucleoside reverse transcriptase inhibitor-containing and protease inhibitor-containing regimens using observational databases. AIDS 15 (9), 1133-1142.

Haase, A.T., Henry, K., Zupancic, M., Sedgewick, G., Faust, R.A., Melroe, H., Cavert, W., Gebhard, K., Staskus, K., Zhang, Z.Q., Dailey, P.J., Balfour, H.H., Erice, A., Perelson, A.S., 1996. Quantitative image analysis of HIV-1 infection in lymphoid tissue. Science 274, 985-989.

Ho, D.D., Neumann, A.U., Perelson, A.S., Chen, W., Leonard, J.M., Markowitz, M., 1995. Rapid turnover of plasma virions and CD4 lymphocytes in HIV-1 infection. Nature 373 (6510), 123-126.

Huang, Y., Rosenkranz, S.L., Wu, H., 2003. Modeling HIV dynamics and antiviral response with consideration of time-varying drug exposures, adherence and phenotypic sensitivity. Math. Biosci. 184, 165-186.

Janeway, C., Travers, P., Walport, M., Shlomchik, M.J., 2001. Immunobiology 5: The Immune System in Health and Disease. Garland Publishing, New York.

Krakovska, O., Wahl, L.M., 2007. Costs versus benefits: best possible and best practical treatment regimens for HIV. J. Math. Biol. 54 (3), 385-406.

Moyle, G., 2003. Protease inhibitor-sparing regimens: new evidence strengthens position. J. Acq. Immun. Def. Synd. 33 (Suppl. 1), 17-25.

Nelson, P.W., Perelson, A.S., 2002. Mathematical analysis of delay differential equation models of HIV-1 infection. Math. Biosci. 171 (1), 73-94.

Nelson, P.W., Murray, J.D., Perelson, A.S., 2000. A model of HIV-1 pathogenesis that includes an intracellular delay. Math. Biosci. 163, 201-215.
Nelson, P.W., Mittler, J.E., Perelson, A.S., 2001. Effect of drug efficacy and the eclipse phase of the viral life cycle on estimates of HIV viral dynamic parameters. J. Acq. Immun. Def. Synd. 26 (5), 405-412.

Nowak, M.A., May, R.M., 2000. Virus Dynamics. Oxford University Press, Oxford.

Perelson, A.S., 2002. Modelling viral and immune system dynamics. Nature Reviews: Immunology 2, 28-36.

Perelson, A.S., Nelson, P.W., 1999. Mathematical analysis of HIV-1 dynamics in vivo. SIAM Rev. 41 (1), 3-44.

Prior, R.W., 2005. Looking around in awareness: playbuilding on HIV-AIDS. Res. Drama Educ. 10 (1), 55-64.

Raguin, G., Chene, G., Morand-Joubert, L., et al., 2004. Puzzle 1 study group. Salvage therapy with amprenavir, lopinavir and ritonavir $200 \mathrm{mg} / \mathrm{d}$ or $400 \mathrm{mg} / \mathrm{d}$ in HIV-infected patients in virological failure. Antiviral Ther. 9, 615-625.

Schacker, T.W., Hughes, J.P., Shea, T., Coombs, R.W., Corey, L., 1998. Biological and virologic characteristics of primary HIV infection. Ann. Intern. Med. 128 (8), 613-620.

Smith, R.J., Wahl, L.M., 2004. Distinct effects of protease and reverse transcriptase inhibition in an immunological model of HIV-1 infection with impulsive drug effects. Bull. Math. Biol. 66 (5), 1259-1283.

Staszewski, S., Dauer, B., Von Hentig, N., et al., 2003a. The LOPSAQ study: 24 week analysis of the double protease inhibitor salvage regimen containing lopinavir plus saquinavir without any additional antiretroviral therapy. In: Second International AIDS Conference, Paris, France, July 2003 (Abstract 853).

Staszewski, S., Dauer, B., Gute, P., et al., 2003b. The CrixiLop cohort study: preliminary results from a salvage study of HIV positive patients treated with indinavir and lopinavir/ritonavir without the addition of reverse transcriptase inhibitors. In: 43rd Interscience Conference on Antimicrobial Agents and Chemotherapy, Chicago, IL, September 2003 (Abstract H-853).

Wahl, L.M., Nowak, M.A., 2000. Adherence and drug resistance: predictions for therapy outcome. Proc. R. Soc. London Ser. B 267, 835-843.

Wu, H., Ding, A., 1999. Population HIV-1 dynamics in vivo: applicable models and inferential tools for virological data from AIDS clinical trials. Biometrics 55 (2), 410-418.

Wu, H., Huang, Y., Acosta, E.P., Park, J.-G., Yu, S., Rosenkranz, S., Kuritzkes, D.R., JEron, J., Perelson, A.S., Gerber, J.G., 2006. Pharmacodynamics of antiretroviral agents in HIV-1 infected patients: using viral dynamic models that incorporate drug susceptibility and adherence. J. Pharmacokinet. Pharmacodyn. 33 (4), 399-419. 